\title{
ABUNDANCE ANALYSIS OF SIX LMC F SUPERGIANTS
}

\author{
ANDREW MCWILLIAM, ROBERT E. WILLIAMS \\ Cerro Tololo Inter-American Observatory \\ Casilla 603 \\ La Serena \\ Chile
}

\begin{abstract}
We have performed an abundance analysis of six Large Magellanic Cloud (LMC) F supergiants, based on infrared photometry and echelle spectra obtained from CTIO. We find a real dispersion in $\mathrm{Fe} / \mathrm{H}$ values, indicating that the $\mathrm{LMC}$ is inhomogeneous. Oxygen abundances appear to be equal in all six stars, resulting in a steep $\mathrm{O} / \mathrm{Fe}$ slope with $\mathrm{Fe} / \mathrm{H}$. Other element abundances appear near normal, although there is a hint of a small enhancement of second s-process peak elements. Iron abundances are probably affected by non-LTE; this does not change our conclusions conceming oxygen.
\end{abstract}

\section{Observations}

Six LMC F supergiants, chosen from Grieve and Madore's list (1986) of non-variable stars, were observed with the CTIO $4 \mathrm{~m}$ telescope and echelle spectrograph with GEC CCD at a resolving power of 18,000 . Typical $\mathrm{S} / \mathrm{N}$ ratios of the final spectra were near 100 . We also obtained infrared $\mathrm{J}$ and $\mathrm{K}$ photometry using the CTIO $1.5 \mathrm{~m}$ telescope and $\mathrm{InSb}$ detector.

\section{Analysis}

Effective temperatures were determined from the V-K, V-J and J-K colours using the calibration of McWilliam (1990). Estimates of the reddening for each star were based on optical and IR photometry and the spectral type assignments. Stellar log g values were computed using an approximate mass found by placing the stars on the theoretical evolutionary tracks of Maeder and Meynet (1988). Model atmospheres were interpolated from the grid of Kurucz (1979), and abundances computed from the measured equivalent widths using an LTE spectrum synthesis program. Microturbulent velocities were found by forcing the computed iron abundances to be independent of equivalent width.

\section{Results}

Table 1 lists metal abundance ratios, $[\mathrm{M} / \mathrm{Fe}]$, relative to the solar abundance distribution. The abundance ratios were taken relative to $\mathrm{Fe}$ I or Fe II lines, depending on the sensitivity of the ratios to uncertainties in the model atmosphere parameters.

Fe I abundances are systematically lower than Fe II abundances by 0.2 dex, probably due to non-LTE. There is a real dispersion in $\varepsilon(\mathrm{Fe})$, indicating an inhomogeneous LMC ISM. The 
oxygen abundance is approximately constant, at $\varepsilon(0)=8.67$ for the six supergiants, resulting in a gradient of $[\mathrm{O} / \mathrm{Fe}]$ with $[\mathrm{Fe} / \mathrm{H}]$ which is much steeper than seen in the Galaxy. Other $\alpha$ elements, such as $\mathrm{Mg}, \mathrm{Si}, \mathrm{Ca}$ and $\mathrm{Ti}$ do not follow the trend of $\mathrm{O} / \mathrm{Fe}$ with $\mathrm{Fe} / \mathrm{H}$. A slight enhancement in the second s-process peak elements would vanish if ratioed with $\mathrm{Fe}$ II abundances, rather than $\mathrm{Fe} I$, as would be more appropriate if Fe I lines are significantly affected by non-LTE.

Table 1. Abundances relative to iron

\begin{tabular}{lrrrrrr}
\hline \hline [M/Fe] & G231 & G274 & G406 & G439 & G538 & G501 \\
\hline & & & & & & \\
\hline C I & -0.54 & -0.16 & -0.32 & -0.44 & -0.52 & -0.78 \\
O I & -0.31 & 0.00 & 0.16 & 0.34 & -0.02 & 0.17 \\
Na I & 0.21 & -0.02 & 0.09 & 0.02 & 0.05 & -0.09 \\
Mg I & -0.01 & 0.01 & -0.10 & -0.08 & -0.13 & -0.10 \\
Si I & 0.55 & 0.28 & 0.36 & 0.32 & 0.38 & 0.32 \\
Ca I & 0.04 & 0.23 & 0.09 & 0.03 & 0.03 & 0.15 \\
Ca II & 0.15 & 0.28 & 0.36 & 0.11 & 0.13 & 0.46 \\
Sc II & -0.04 & 0.01 & -0.10 & -0.12 & -0.10 & -0.20 \\
Ti I & 0.12 & 0.07 & 0.15 & -0.06 & 0.23 & 0.28 \\
Ti II & 0.05 & 0.29 & 0.22 & 0.27 & 0.37 & 0.34 \\
V I & -0.41 & -0.47 & -0.70 & -0.73 & -0.64 & $\ldots$ \\
V II & 0.01 & 0.10 & 0.01 & 0.20 & -0.22 & $\ldots$ \\
Cr I & -0.20 & -0.05 & -0.19 & -0.03 & -0.05 & -0.33 \\
Cr II & 0.02 & 0.11 & 0.06 & 0.04 & 0.03 & 0.03 \\
Mn I & -0.12 & -0.33 & -0.41 & -0.32 & -0.35 & -0.47 \\
Mn II & $\ldots$ & -0.52 & -0.51 & $\ldots$ & -0.57 & -0.71 \\
Co I & -0.99 & -1.02 & -1.10 & -0.52 & -0.89 & -0.53 \\
Ni I & 0.00 & -0.12 & -0.14 & -0.29 & -0.17 & -0.12 \\
Zn I & -0.18 & -0.51 & -0.19 & -0.33 & -0.27 & 0.00 \\
Y II & -0.03 & -0.08 & 0.10 & -0.02 & 0.11 & 0.02 \\
Zr II & -0.06 & 0.14 & 0.04 & 0.16 & 0.25 & -0.34 \\
Ba II & 0.41 & 0.19 & 0.29 & 0.45 & -0.06 & 0.32 \\
La II & 0.10 & 0.20 & 0.33 & 0.38 & 0.16 & 0.25 \\
Ce II & 0.16 & 0.47 & 0.38 & 0.51 & 0.46 & 0.43 \\
Pr II & $\ldots$ & 0.09 & 0.19 & 0.12 & 0.28 & 0.16 \\
Nd II & 0.19 & 0.34 & 0.32 & 0.37 & 0.38 & 0.28 \\
Sm II & 0.16 & 0.21 & 0.38 & 0.42 & 0.30 & 0.25 \\
Gd II & -0.83 & -0.41 & -0.30 & -0.26 & -0.34 & $\ldots$ \\
Dy II & $\ldots$ & 0.53 & 0.00 & 0.34 & 0.28 & 0.05 \\
Hf II & 0.43 & 0.31 & 0.57 & 0.79 & 0.14 & 0.08 \\
& & & & & & \\
[Fe I/II] & -0.27 & -0.44 & -0.62 & -0.68 & -0.42 & -0.62 \\
[Fe II/II] & -0.05 & -0.28 & -0.34 & -0.58 & -0.18 & -0.33 \\
& & & & & &
\end{tabular}

\section{References}

Grieve, G.R. and Madore, B.F. (1986), Astrophys. J. 62, 427.

Kurucz, R.L. (1979), Astrophys. J. Suppl. Ser. 40, 1.

Maeder, A. and Meynet, G. (1988), Astron. Astrophys. Suppl. Ser. 76, 411.

McWilliam, A. (1990), Astron. J. submitted. 